“(C) 2015 IEEE. Personal use of this material is permitted. Permission from IEEE must be obtained for all other uses, in any current or future media, including reprinting/republishing this material for advertising or promotional purposes, creating new collective works, for resale or redistribution to servers or lists, or reuse of any copyrighted component of this work in other works." 


\title{
A Multi-stage Design Framework for the Development of Task-specific Robotic Exoskeletons
}

\author{
Marc G. Carmichael, Richardo Khonasty, Dikai Liu
}

\begin{abstract}
This work presents a multi-stage design framework for developing robotic exoskeletons suited for specific tasks, such as individualized exercises that meet the needs of patients undergoing physical therapy. The framework systematically develops the exoskeleton based on the required task space, represented by a set of limb poses which may be defined directly, or indirectly using means such as motion capture. The design process seeks to maximize the poses inside and surrounding the defined task space whilst ensuring additional criteria required to perform the task are satisfied. A case study demonstrates the framework applied to develop two variations of shoulder exoskeleton suited for two specific upper limb activities. Prototype exoskeletons based on the framework's outcomes were constructed, and their suitability for use in their intended tasks were evaluated.
\end{abstract}

\section{INTRODUCTION}

Robotic exoskeletons are used to assist and administer therapies to persons with upper limb impairments [1], [2], [3], [4]. Although such devices are designed with a kinematic structure similar to that of the human upper limb, the requirements of avoiding human-robot collisions, robot self collisions and kinematic singularities means that the workspace in which the exoskeleton can operate effectively is often smaller than the natural range of motion (ROM) of the human upper limb. Hence the design of robotic exoskeletons often focuses on the limb motion that can be achieved, attempting to allow functional operation in a prioritized workspace within the limb's ROM [2], [5], [6], [7], or maximizing the total workspace that can be reached when the device is worn [8], [9], [10].

Despite having an operating range smaller than that of the human upper limb, exoskeletons are effective rehabilitation devices as long as suitable exercises can be performed. The optimal therapy to administer depends on the individual needs of the patient, thus motivating the development of patient-specific exoskeletons designed for the therapies that would maximize a patient's recovery. This work presents a design framework for developing robotic exoskeletons suited for specific tasks. A multi-stage approach is proposed as it reduces the design process into individualized steps, allowing the multiple and often conflicting design objectives to be addressed in a systematic manner. The framework firstly attempts to develop the exoskeleton such that all limb poses required during the task are reachable. If this is achievable, subsequent stages seek to further improve the

M. G. Carmichael, R. Khonasty and D. Liu are with the Centre for Autonomous Systems, University of Technology Sydney (UTS), Australia. E-mail: marc.carmichael@uts.edu.au

This work was supported by the Australian Research Council (ARC) Linkage Grant (LP140100950), and Burwell Technologies Pty Ltd. design, for example by allowing neighbouring limb poses to be reached, or optimizing some other criteria so as to improve performance of the task. This systematic approach facilitates the development of exoskeletons that satisfy a number of requirements, whilst maximizing the region about the desired task that the robot can operate in. Section II presents the task-specific multi-stage design framework. Section III then presents a case study demonstrating the framework being utilized to design two variations of exoskeleton, which are then constructed and evaluated.

\section{Multi-Stage Design Framework}

To develop the framework we first generalize the problem of developing an exoskeleton for specific tasks. For clarity we state some definitions used in this work. Firstly, even though the patient may have a limited range of motion due to physical injury, when we refer to $R O M$ it is in reference to the normative ROM of a healthy person, since achieving a healthy ROM is ultimately the objective of utilizing the exoskeleton for rehabilitation. Secondly, the term ROM is with respect to the parts of the human body which will be wearing the device being designed, which in this work is the upper limb.

A pose of the patient's limb that will wear the exoskeleton is represented by vector $\mathbf{p}$ of generalized coordinates. These coordinates may be local to the body (e.g. limb coordinates such as joint flexion or extension) or expressed in a global frame. Either way, vector $\mathbf{p}$ fully defines one pose of the limb and is bounded within space $\psi_{H}$ corresponding to the limb's ROM. The operator's ROM is quantized with an appropriate resolution to produce a finite set of limb poses distributed throughout the limb's ROM, represented as $\psi_{H}=\left\{\mathbf{p}_{1}, \cdots, \mathbf{p}_{n}\right\}$. An even distribution is required so as to avoid skewing the design process into favoring regions with a higher density, assuming this is undesired.

The task to be performed requires the patient to maneuver their limb throughout a subspace of their limb's ROM. Hence, for the exoskeleton to be suitable for the task, it must allow the operator to maneuver their limb throughout this entire subspace while the device is worn. This task-space is represented by set $\psi_{T}$ and is a subset of $\psi_{H}$. Figure 1 shows abstract representations of two proposed methods for defining $\psi_{T}$ :

1) Bounds of a region within the patient's ROM that needs to be reached are first defined, and elements of $\psi_{H}$ existing within this region are collected to form $\psi_{T}$ (Fig. 1a). 


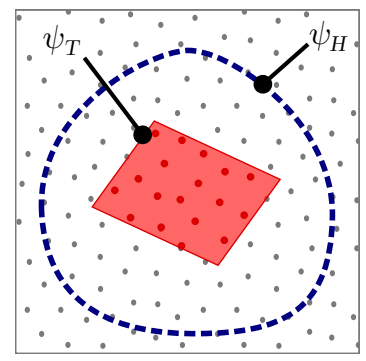

(a)

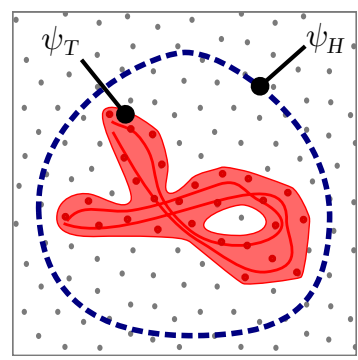

(b)
Fig. 1. Abstract representations of methods for defining task space $\psi_{T}$ within the limb's ROM defined by $\psi_{H}$. Each dot represents a unique $\mathbf{p}$ vector used to define limb pose. (a) Set $\psi_{T}$ created by directly defining boundaries within the limb's ROM. (b) Set $\psi_{T}$ created by using motion capture to collect nearest matching poses.

2) Limb poses required during the task are measured, e.g. using motion capture, then are down-sampled by assigning each to its closest matching pose in $\psi_{H}$, with matching poses collected to form $\psi_{T}$ (Fig. 1b).

For the patient to perform a task as required, it is not simply enough to be capable of maneuvering their limb across all of $\psi_{T}$. Other criteria dependent on the design of the exoskeleton must also be satisfied. Examples may include; valid inverse kinematic solutions, no kinematically singular positions, no collisions between human and robot, and a minimum end-effector payload capacity. These criteria are represented by $k$ generalized expressions $c_{i}$ (1) which we define as equalling zero when the criterion is satisfied.

$$
\underset{i \in[1, k]}{c_{i}}= \begin{cases}0 & \text { if criterion } i \text { is satisfied } \\ 1 & \text { if criterion } i \text { is not satisfied }\end{cases}
$$

It is assumed that the exoskeleton has a finite number of design variables which can be adjusted. Design variables used in this work are limited to the kinematics of the exoskeleton. For convenience these design variables are represented as a set $D V=\left\{a_{1}, a_{2} \cdots, a_{n}\right\}$. The objective of the exoskeleton design process then becomes finding a set of design variables $D V$ such that the desired task (represented by $\psi_{T}$ ) can be performed, subject to all additional requirements (criteria $c_{1}$ to $c_{k}$ ) being satisfied.

From this generalization a framework for developing taskspecific exoskeletons is proposed. The design process, illustrated in Fig. 2, utilizes several stages to optimize multiple yet often competing design objectives. Each of the stages are described in Sections II-A to II-C.

\section{A. Design Stage 1}

A logical first objective is to optimize the exoskeleton such that the patient can reach as much of task space $\psi_{T}$ as possible, whilst ensuring all other required criteria are satisfied. A cost function for this objective is derived by constructing the set of all limb poses within $\psi_{T}$ for which all criteria $c_{1}$ to $c_{k}$ are satisfied. We refer to this as the performable subset of poses and represent it as $\psi_{P}(2)$. It

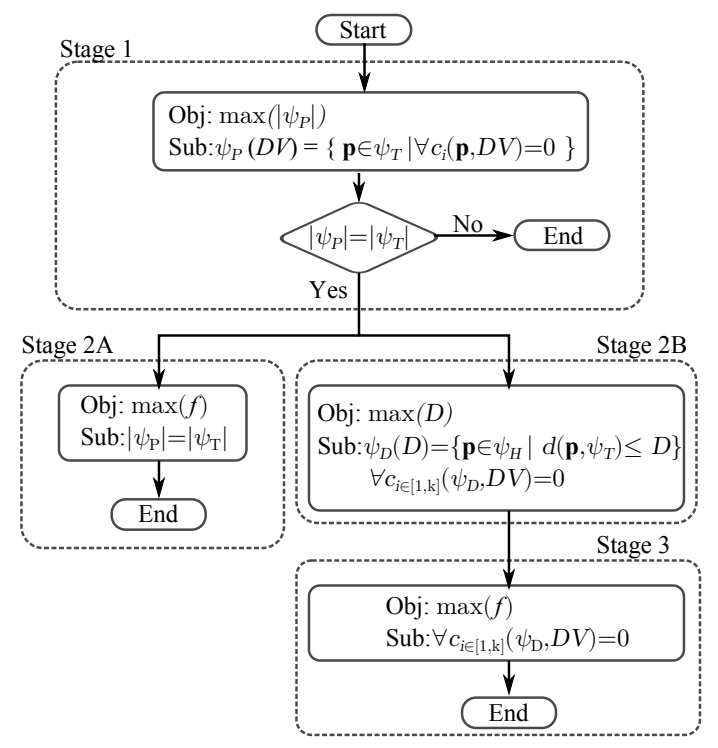

Fig. 2. Multi-stage design framework flowchart.

is noted that $\psi_{P}$ is a function of $D V$ since the choice of design variables affects if $c_{1}$ to $c_{k}$ are satisfied.

$$
\psi_{P}(D V)=\left\{\mathbf{p} \in \psi_{T} \mid \underset{i \in[1, k]}{\forall c_{i}}(\mathbf{p}, D V)=0\right\}
$$

The objective is to maximize the size of set $\psi_{P}$ (3) which can be solved computationally using existing optimization techniques. Since $\psi_{P} \subseteq \psi_{T}$, the optimal result will be $\left|\psi_{P}\right|=$ $\left|\psi_{T}\right|$, or put simply, all poses in the defined task space $\psi_{T}$ can be reached by the exoskeleton with all additional criteria satisfied.

$$
\text { Stage } 1 \text { objective }: \max \left(\left|\psi_{P}\right|\right)
$$

The outcome of the Stage 1 optimization determines how the design process proceeds. Consider that the optimal result $\left(\left|\psi_{P}\right|=\left|\psi_{T}\right|\right)$ may not be unique to a single set of design variables. Hence, if the optimal result is obtained in Stage 1, the exoskeleton design process continues to Stage 2 as further improvement may be possible. Alternatively if the optimal result was not achieved $\left(\left|\psi_{P}\right|<\left|\psi_{T}\right|\right)$ the design process finishes as it's assumed no further improvement can be made within the presented framework. Additional analysis may be performed to determine if the non-optimal design outcome of Stage 1 is satisfactory for the desired application.

\section{B. Design Stage 2}

Given that the result from Stage 1 may undergo further improvement, the framework provides the designer with a choice as to the direction in which further design improvement should be sought. This choice is to either attempt to optimize exoskeleton performance while maintaining a certain number of reachable limb positions, or maximizing the number of reachable limb positions subject to maintaining a certain level of performance. The choice of approach 
taken is left to the designer, and should be made taking into consideration the desired application.

The first direction (Stage 2A) is to optimize additional criteria, subject to all the limb poses in the task space still being performable. This option may be chosen in situations where optimizing other criteria is beneficial (e.g. manipulability, overall size, etc), but reaching limb poses outside of those defined in $\psi_{T}$ is not considered as beneficial. The objective is generalized as function $f$ having a scalar output relating to some performance measure that the designer seeks to maximize. For example the optimization may maximize the exoskeleton's manipulability averaged over all limb poses within $\psi_{T}$. Once the solution for the Stage 2A optimization is found (4), the design procedure ends as it is assumed no further improvement can be made.

$$
\begin{aligned}
& \text { Stage 2A objective }: \max (f) \\
& \text { Subject to : }\left|\psi_{P}\right|=\left|\psi_{T}\right|
\end{aligned}
$$

The second direction (Stage 2B) is to further optimize the exoskeleton such that limb poses surrounding those in the task space $\psi_{T}$ are also performable. This option may be chosen in situations where developing an exoskeleton suitable for a broader range of applications is beneficial, allowing limb poses outside of those initially defined in $\psi_{T}$ to be performed. One implementation could be to maximize the number of poses outside of $\psi_{T}$ that are performable, however this approach does not prioritize the poses in any way and hence many poses that become performable may be unlikely adopted during the task. In the absence of additional information that may be used to prioritize limb poses outside of $\psi_{T}$, we propose that it is logical to prioritize them based on their distance to poses initially defined in $\psi_{T}$ so that any additional performable poses have limb configurations similar to those expected to be adopted during the task.

Using a suitable metric that quantifies the distance between two limb poses, a function that calculates the minimum distance between an arbitrary limb pose $\mathbf{p}$ and its closest pose in a set $\psi$ can be created (5). The metric chosen depends on the coordinates used to define limb pose, and hence consideration should be taken when creating this distance function.

$$
\text { minimum distance }=d(\mathbf{p}, \psi)
$$

A margin surrounding the poses in task space $\psi_{T}$ is defined using scalar distance value $D$. All poses in the limb's ROM set $\psi_{H}$ are checked for their distance to the closest pose in set $\psi_{T}$ and if are found to be closer than margin $D$ they are put into a set defined as $\psi_{D}(6)$. This process is illustrated in Fig. 3 b.

$$
\psi_{D}(D)=\left\{\mathbf{p} \in \psi_{H} \mid d\left(\mathbf{p}, \psi_{T}\right) \leq D\right\}
$$

The objective then is to find the design variables $D V$ that maximize the margin $D$ subject to all poses within $\psi_{D}$ satisfying the criteria $c_{1}$ to $c_{k}$ (7). Once the solution for

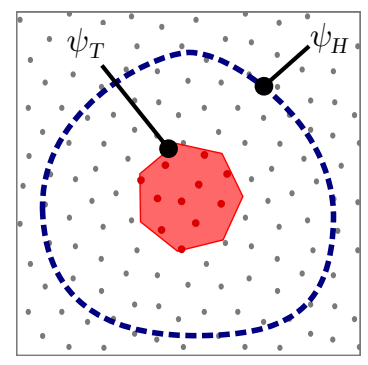

(a)

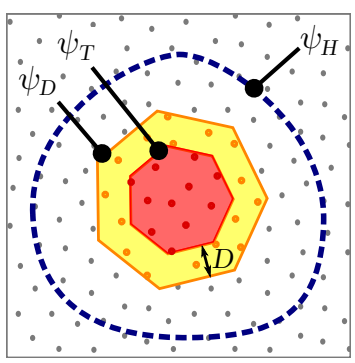

(b)
Fig. 3. Illustrative comparison of the two options available to improve the exoskeleton design in Stage 2. (a) Increase exoskeleton performance without seeking to allow poses outside of $\psi_{T}$ to be performable. (b) Increase the margin surrounding $\psi_{T}$ within which all poses are performable.

the Stage 2B optimization is found the design procedure continues to Stage 3.

$$
\begin{aligned}
& \text { Stage 2B objective : } \max (D) \\
& \text { Subject to : } \underset{i \in[1, k]}{\forall c_{i}}\left(\psi_{D}, D V\right)=0
\end{aligned}
$$

\section{Design Stage 3}

Although the previous stage maximized the margin surrounding $\psi_{T}$ within which all poses satisfy all design criteria, there may still be scope to improve the design by optimizing some performance criteria. Similar to the case with Stage $2 \mathrm{~A}$, an objective function $f$ is defined with a scalar output relating to a performance measure that the designer seeks to maximize. The third and final stage optimization is applied to this objective function, subject to all of poses within set $\psi_{D}$ (based on the maximum margin $D$ obtained in Stage 2B) still being performable (8). Once the optimal solution is found, the design procedure is complete.

$$
\begin{array}{r}
\text { Stage } 3 \text { objective }: \max (f) \\
\text { Subject to : } \underset{i \in[1, k]}{\forall c_{i}}\left(\psi_{D}, D V\right)=0
\end{array}
$$

\section{CASE Study}

The following case study demonstrates the framework being used to design the 3-DOF shoulder mechanism of an upper limb exoskeleton. Some aspects of the design process such as the robot's forward and inverse kinematics, as well as the calculation of human-robot collisions and kinematic singularity are similar to previous work that optimized an exoskeleton using a single-stage process [10]. Readers are directed to this previous work for these specifics.

In this case study two different exoskeleton designs are developed based on two different tasks. Each exoskeleton was optimized using the framework proposed, manufactured based on the design results, and preliminary experiments performed to validate their suitability in performing their respective task. 


\section{A. Exoskeleton to be developed}

The exoskeleton utilizes three joints interconnected with curved links to form a spherical joint with its center of rotation located approximately at the center of the patient's shoulder. The base (link 0) is assumed to be fixed relative to the patient's torso, and the link distal of the third joint (link 3) is assumed to be physically attached to the patient's arm.

The design variables to be optimized include the bends in links 1, 2 and 3, parameterized as $\alpha_{1}, \alpha_{2}$, and $\alpha_{3}$ respectively. Link 0 determines the orientation of the first joint relative to the torso, parameterized by two successive rotations $\alpha_{0 x}$ and $\alpha_{0 y}$ about the torso $x$ and $y$ axes. A final parameter $\Omega$ is used to ensure unique inverse kinematic solutions are utilized. These design variables are defined as a set $D V=\left\{\alpha_{0 x}, \alpha_{0 y}, \alpha_{1}, \alpha_{2}, \alpha_{3}, \Omega\right\}$. More information on the exoskeleton and its design variables are in [10].

\section{B. Definition of human ROM and desired tasks}

Since the exoskeleton being developed encompasses the patient's shoulder, we define the limb's ROM as a set of unit quaternions representing the orientation of the upper arm relative to the torso. Set $\psi_{H}$ of human shoulder ROM is created by first generating a finite set of quaternions evenly distributed across all SO3 rotational space, then using a biomechanical model of the upper limb [11] quaternions corresponding to poses which can be reached by the human shoulder are collected to form set $\psi_{H}$.

The two tasks for which exoskeletons are being developed are; 1) drinking, and 2) reaching behind the lower back. These different activities were chosen as they are both typical upper limb motions, yet require the subject to adopt significantly different arm poses. To define the task space required for each task, motions of the upper limb are recorded using a Xsens MVN motion capture system whilst a subject performs the activity, as shown in Fig. 4a. The motion is repeated several times to obtain variations in the adopted poses. Quaternions representing the orientation of the upper arm are extracted from the recorded motion, down-sampled according to the closest matching quaternion in set $\psi_{H}$, and collected to form two sets $\psi_{T}$ representing the limb poses required to perform each of the two tasks.

\section{Additional criteria to be satisfied}

As well as reaching the entire task space, three additional criteria are defined which need to be satisfied:

Criterion $c_{1}$ - At each limb pose, a valid solution for the exoskeleton's inverse kinematics must exist

Criterion $c_{2}$ - At each limb pose, the exoskeleton must not be in collision with the patient

Criterion $c_{3}$ - At each limb pose, the exoskeleton must not be near a kinematically singular condition

Computation of each of these criterion is identical to that implemented in previous work [10]. Criterion $c_{1}$ is tested by calculating the inverse kinematic solution of the mechanism

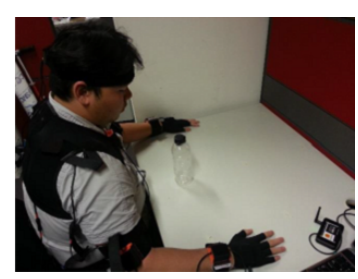

(a)

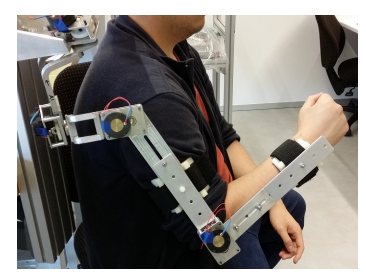

(b)
Fig. 4. (a) Recording upper limb motion during tasks using Xsens MVN motion capture system. (b) Upper limb exoskeleton developed by the multistage development framework.

according to the limb's orientation. Criterion $c_{2}$ is tested using a geometric representation of the exoskeleton and patient to check if collisions are occurring. Criterion $c_{3}$ is tested by comparing the square root of the smallest eigenvalue of the mechanism's Jacobian matrix to a threshold value to determine if the mechanism is too close to singularity.

\section{Optimization results}

The multi-stage design procedure was implemented in MATLAB. Each optimization stage utilized a genetic algorithm having a randomly generated initial population of size 500. The design procedure was conducted using two different sets of $\psi_{T}$ representing the two tasks, resulting in two different exoskeleton designs. Each design process was repeated several times to ensure that the best possible solution was found.

The motions in the two tasks required the arm to span a relatively small region of the shoulder's ROM, hence both designs achieved the optimal solution during Stage 1 and proceeded to Stage 2. The Stage 2B approach which maximized the number of performable limb poses neighbouring those in the task space was chosen. The metric used to define distance between limb pose was the size of the relative rotation between the two quaternions. Stage 3 had the objective of increasing the manipulability of the mechanism for limb poses outside of the margin $D$ obtained from Stage $2 \mathrm{~B}$. This was implemented using a cost function that summed the singularity conditions for each pose outside of $\psi_{D}$, with each being weighted based on their distance to the nearest pose in $\psi_{D}$. The optimal design variables obtained at the conclusion of the three stage development process for both exoskeletons are shown in Table I.

TABLE I

RESULTS FROM THE MULTI-STAGE DESIGN PROCESS

\begin{tabular}{|c|c|c|c|c|c|}
\cline { 2 - 6 } \multicolumn{1}{c|}{} & \multicolumn{5}{c|}{ Design Variables $\left[\mathrm{Deg}^{\mathrm{O}}\right]$} \\
\hline Task: & $\alpha_{0 x}$ & $\alpha_{0 y}$ & $\alpha_{1}$ & $\alpha_{2}$ & $\alpha_{3}$ \\
\hline A) Drinking & 8.5 & 26.1 & 77.9 & 80.2 & -0.02 \\
\hline B) Lower back reaching & -44.3 & 37.8 & 71.8 & 78.1 & -0.01 \\
\hline
\end{tabular}

\section{E. Preliminary Experiments}

A preliminary experiment was conducted with 5 healthy participants, each rating two exoskeleton prototypes built using the design variables shown in Table I. The exoskeletons 
were worn while different motions were performed, including the two motions that the exoskeletons were specifically developed for. Participants were asked to rate how easy the task could be performed, with 0 representing that the motion was unable to be conducted, and 10 representing that the motion felt natural with very little to no hindrance from the exoskeleton. The exoskeletons were not powered for the test, and participants were not made aware of which motions the exoskeletons were developed for. Five different motions were performed to make it less obvious as to which motion each exoskeletons was specifically designed for. Experiments were approved by the UTS Human Research Ethics Committee.

Feedback from the participants indicated that both exoskeletons had a wide range of capability, able to perform most of the motions asked of them. Some participants were able to perform tasks that others could not. Figure 5 shows the results with respect to the drinking and the reaching your lower back tasks the exoskeletons were designed for. It is seen that in both tasks, the preferred exoskeleton was the one that was designed specifically for that task.

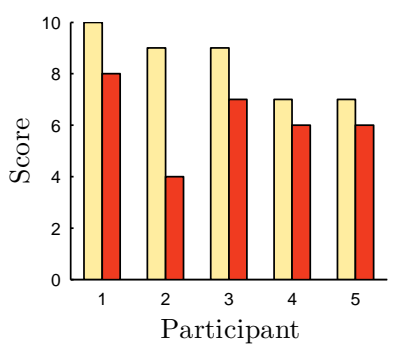

(a) Drinking

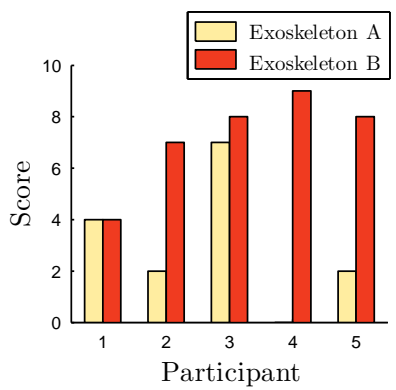

(b) Reaching lower back
Fig. 5. Feedback from five participants as to how suited each exoskeleton felt for motions (a) drinking, and (b) reaching lower back. Participants gave each exoskeleton a score from 0 to 10 , with 10 representing that the motion felt natural with little hinderance, and 0 representing the motion was awkward and unable to be performed. Results indicate Exoskeleton A (designed specifically for the drinking motion) was preferred for that motion compared to Exoskeleton B; and likewise Exoskeleton B (designed specifically for the lower back motion) was preferred for that motion compared to Exoskeleton A.

\section{Discussion}

The preliminary experiments suggest that the multi-stage design framework was able to perform as intended, developing two different exoskeleton designs that were suited to their intended tasks. The variation in scores given to each exoskeleton suggests that factors other than the design variables developed in the case study affected how the participants perceived each exoskeleton's suitability for the task. We theorize that one source for this variation may come from natural variations in limb motion that participants employ when performing tasks. Such variation may be accommodated by the multi-stage design framework by utilizing motion capture data obtained directly from the specific user. Although experiments were comprised of a small number of participants and tasks, the two exoskeletons were shown to be preferred in the tasks they were specifically developed for, encouraging further research.

\section{Conclusions}

The framework presented in this paper provides a method of developing exoskeletons designed for specific tasks through a multi-stage design procedure. The framework provides the means of optimizing both the limb poses that the exoskeleton is able to achieve, while also optimizing additional criteria that may required for the exoskeleton to perform the task. We demonstrated the framework in a case study developing two exoskeleton designs suited for two different upper limb tasks. The resulting designs allowed all required limb poses to be achieved, whilst also being optimized with respect to additional criteria relating to human-robot collision and singularity conditions. Prototypes of the exoskeletons were then manufactured and utilized in preliminary tests. Results indicated that the exoskeletons were preferred in the tasks they were designed for, validating the proposed framework. It is intended that the presented framework will help facilitate the development of task-specific exoskeletons capable of administering patientspecific therapies.

\section{REFERENCES}

[1] M. Mihelj, T. Nef, and R. Riener, "ARMin - toward a six DoF upper limb rehabilitation robot," in Biomedical Robotics and Biomechatronics, 2006. BioRob 2006. The First IEEE/RAS-EMBS International Conference on, pp. 1154-1159, Feb 2006.

[2] M. Mihelj, T. Nef, and R. Riener, "ARMin II - 7 DoF rehabilitation robot: mechanics and kinematics," in Robotics and Automation, 2007 IEEE International Conference on, pp. 4120-4125, April 2007.

[3] Y. Mao and S. Agrawal, "Design of a cable-driven arm exoskeleton (CAREX) for neural rehabilitation," Robotics, IEEE Transactions on, vol. 28, pp. 922-931, Aug 2012.

[4] T. Rahman, W. Sample, R. Seliktar, M. Scavina, A. Clark, K. Moran, and M. Alexander, "Design and testing of a functional arm orthosis in patients with neuromuscular diseases," Neural Systems and Rehabilitation Engineering, IEEE Transactions on, vol. 15, pp. 244-251, June 2007.

[5] J. Perry, J. Rosen, and S. Burns, "Upper-limb powered exoskeleton design," Mechatronics, IEEE/ASME Transactions on, vol. 12, pp. 408417, Aug 2007.

[6] J. Rosen, J. Perry, N. Manning, S. Burns, and B. Hannaford, "The human arm kinematics and dynamics during daily activities - toward a 7 dof upper limb powered exoskeleton," in Advanced Robotics, 2005. ICAR '05. Proceedings., 12th International Conference on, pp. 532539, July 2005.

[7] C. Carignan, M. Liszka, and S. Roderick, "Design of an arm exoskeleton with scapula motion for shoulder rehabilitation," in Advanced Robotics, 2005. ICAR '05. Proceedings., 12th International Conference on, pp. 524-531, 2005.

[8] F. Martinez, I. Retolaza, A. Pujana-Arrese, A. Cenitagoya, J. Basurko, and J. Landaluze, "Design of a five actuated dof upper limb exoskeleton oriented to workplace help," in Biomedical Robotics and Biomechatronics, 2008. BioRob 2008. 2nd IEEE RAS \& EMBS International Conference on, pp. 169-174, 2008.

[9] H. S. Lo and S. Xie, "Optimization of a redundant $4 \mathrm{R}$ robot for a shoulder exoskeleton," in Advanced Intelligent Mechatronics (AIM), 2013 IEEE/ASME International Conference on, pp. 798-803, 2013.

[10] M. G. Carmichael and D. Liu, "Human biomechanical model based optimal design of assistive shoulder exoskeleton," in Field and Service Robotics (L. Mejias, P. Corke, and J. Roberts, eds.), vol. 105 of Springer Tracts in Advanced Robotics, pp. 245-258, Springer International Publishing, 2014.

[11] J. Lenarcic and A. Umek, "Simple model of human arm reachable workspace," Systems, Man and Cybernetics, IEEE Transactions on, vol. 24, pp. 1239 -1246, Aug 1994. 